\title{
Visualisation of coronary venous anatomy by computed tomography angiography prior to cardiac resynchronisation therapy implantation
}

\author{
U. C. Nguyên 1,2 • M. J. M. Cluitmans² · J. G. L. M. Luermans² · M. Strik² · C. B. de Vos² • B. L. J. H. Kietselaer2,3 • \\ J. E. Wildberger ${ }^{3} \cdot$ F. W. Prinzen ${ }^{1} \cdot$ C. Mihl ${ }^{3} \cdot$ K. Vernooy ${ }^{2,4}$
}

Published online: 20 July 2018

(c) The Author(s) 2018

\begin{abstract}
Background The purpose of this study was to illustrate the additive value of computed tomography angiography (CTA) for visualisation of the coronary venous anatomy prior to cardiac resynchronisation therapy (CRT) implantation.

Methods Eighteen patients planned for CRT implantation were prospectively included. A specific CTA protocol designed for visualisation of the coronary veins was carried out on a third-generation dual-source CT platform. Coronary veins were semi-automatically segmented to construct a 3D model. CTA-derived coronary venous anatomy was compared with intra-procedural fluoroscopic angiography (FA) in right and left anterior oblique views.

Results Coronary venous CTA was successfully performed in all 18 patients. CRT implantation and FA were performed in 15 patients. A total of 62 veins were visualised; the number of veins per patient was 3.8 (range: 2-5). Eighty-five per cent (53/62) of the veins were visualised on both CTA and FA, while 10\% (6/62) were visualised on CTA only, and 5\% (3/62) on FA only. Twenty-two veins were present on the lateral or inferolateral wall; of these, $95 \%$ (21/22) were visualised by CTA. A left-sided implantation was performed in 13 patients, while a right-sided implantation was performed in the remaining 2 patients because of a persistent left-sided superior vena cava with no left innominate vein on CTA.

Conclusion Imaging of the coronary veins by CTA using a designated protocol is technically feasible and facilitates the CRT implantation approach, potentially improving the outcome.
\end{abstract}

Keywords Coronary veins $\cdot$ Computed tomography angiography $\cdot$ Fluoroscopic angiography $\cdot$ Cardiac resynchronisation therapy

\section{What's new?}

Electronic supplementary material The online version of this article (https://doi.org/10.1007/s12471-018-1132-2) contains supplementary material, which is available to authorized users.

$\triangle$ U. C. Nguyên

u.nguyen@maastrichtuniversity.nl

1 Department of Physiology, Cardiovascular Research Institute Maastricht (CARIM), Maastricht University Medical Centre (MUMC+), Maastricht, The Netherlands

2 Department of Cardiology, Cardiovascular Research Institute Maastricht (CARIM), Maastricht University Medical Centre (MUMC+), Maastricht, The Netherlands

3 Department of Radiology \& Nuclear Medicine, Cardiovascular Research Institute Maastricht (CARIM), Maastricht University Medical Centre (MUMC+), Maastricht, The Netherlands

4 Department of Cardiology, Radboud University Medical Centre, Nijmegen, The Netherlands
- Coronary venous anatomy is visualised prior to cardiac resynchronisation therapy (CRT) using a designated computed tomography angiography (CTA) protocol.

- A high concordance (85\%) in coronary venous anatomy between CTA and fluoroscopic angiography was found.

- Coronary venous CTA additionally impacted the CRT implantation approach in a quarter of the patients, showing potential for the clinical workup in CRT.

\section{Introduction}

Despite the effectiveness of cardiac resynchronisation therapy (CRT), one-third of eligible patients fail to benefit [1]. Placing the left ventricular (LV) lead in an appropriate position in late activated myocardium remote from scarring is a prerequisite for a response to $\operatorname{CRT}[2,3]$. The majority of studies investigating LV-lead placement 
have utilised echocardiography [3] and cardiac magnetic resonance imaging (CMR) [4] to define a LV-lead target segment pre-procedurally. The LV lead was subsequently guided to a coronary vein in the target segment using fluoroscopic angiography (FA). However, the practical issue remains that a target segment cannot be reached intra-procedurally, as not all LV segments are covered by coronary venous anatomy. A recent imaging-guided CRT study demonstrated that in half of the patients, no coronary vein was present in the pre-defined CMR or echocardiographic based target segment. Coronary venous anatomy is highly variable regarding trajectory, shape and number of veins $[5,6]$. Imaging is therefore essential and can be used to tailor LV-lead placement by pre-defining a target vein or even considering alternative biventricular pacing options if suboptimal conditions prevail.

FA is most commonly used for visualisation of the coronary veins but is invasive, thus prohibiting pre-procedural anticipation of patient-specific anatomy. Computed tomography angiography (CTA) may enable three-dimensional (3D) non-invasive visualisation of the coronary veins and could be performed prior to CRT implantation.

The purpose of this study was to illustrate the additive value of visualisation of the coronary venous anatomy by CTA prior to CRT implantation by (1) elucidating on clinical cases and by (2) comparing the coronary venous anatomy on CTA with FA.

\section{Methods}

\section{Study population}

Patients referred for a CRT device implantation were prospectively enrolled from April to December 2017 at the Maastricht University Medical Centre. Exclusion criteria were standard contra-indications for CTA including severe renal insufficiency, contrast media allergy and claustrophobia. The institutional Review Board approved the study protocol and patients gave written consent for the use of their data.

\section{Pre-procedural computed tomography angiography}

CTA images were acquired up to 1 month prior to the scheduled CRT implantation. Scans were performed using a third-generation dual-source CT scanner (Somatom Definition Force, Siemens Healthineers, Forchheim, Germany) with a $2 \times 192 \times 0.6 \mathrm{~mm}$ slice collimation; reference tube voltage was set to $100 \mathrm{kV}_{\text {ref }}$ using automated tube voltage selection (CARE kV); and a reference quality tube current of $400 \mathrm{mAs}$ using tube current modulation software (CareDose 4D). The gantry rotation time was $250 \mathrm{~ms}$ and data were acquired with retrospective gating (pitch: 0.15). Image reconstruction was done with an individually adapted field of view at $0.6 \mathrm{~mm}$ slice thickness with an increment of $0.4 \mathrm{~mm}$ using a Bv40 kernel and iterative reconstruction (ADMIRE; strength 3). A test bolus was injected at the level of the coronary sinus (CS) or ascending aorta to assess optimal delay for the administration of contrast medium (CM). Delay was calculated by time-to-peak +10 or $+20 \mathrm{~s}$ for the $\mathrm{CS}$ or ascending aorta, respectively. Patients received $72 \mathrm{ml}$ pre-warmed Iopromide 300 (Ultravist, Bayer Healthcare, Berlin, Germany) at a flow rate of $6 \mathrm{ml} / \mathrm{s}$ followed by an 84-ml mixed bolus (20\% CM/80\% saline) and a 40-ml saline flush at a flow rate of $3 \mathrm{ml} / \mathrm{s}$. Total iodine load was $26.6(21.6+5) \mathrm{g}$. Injection pressure and total amount of CM were monitored by a dedicated information platform (Certegra Informatics Solution, Bayer). Coronary venous anatomy was evaluated under supervision by a radiologist with 7 years' experience in cardiac CTA. 3D reconstructions of the epicardium and coronary veins were constructed using a semi-automatic segmentation approach (Seg3D v2.4, SCI, University of Utah, USA). A 3D cardiac model comprising the coronary veins and epicardium was interactively shown to the implanting physician prior and during CRT implantation to guide LV-lead placement.

\section{Intra-procedural fluoroscopic angiography}

Coronary venous anatomy was evaluated intra-procedurally by an occlusive FA after placement of the right atrial and right ventricular (RV) lead. The CS was cannulated using a CS guiding catheter (Medtronic, Minneapolis, MN, USA) under FA. After cannulation of the CS, a balloon catheter (Medtronic) was advanced towards the proximal site of the $\mathrm{CS}$ for occlusion. Once the balloon catheter was in a stable position, fluoroscopic angiograms of the coronary veins were recorded in right anterior oblique (RAO) and left anterior oblique (LAO) views (Inifinix-I, Toshiba Medical, Ottawara, Tochigi, Japan). The coronary venous anatomy from FA was evaluated together with the implanting cardiologist. After acquisition of the fluoroscopic angiograms, the balloon catheter was withdrawn from the CS and a quadripolar or bipolar LV lead was placed in a coronary vein guided by FA. An electrode with a low voltage threshold without phrenic nerve stimulation and long electrical delay was selected for pacing.

\section{Data analyses}

Continuous variables are expressed as mean \pm standard deviation and dichotomous variables in frequencies. The coronary venous angiogram from both CTA and FA was classified according to the American Heart Association 17-segment heart model (Fig. 1). The CS tributaries from the 3D 
Fig. 1 Coronary venous classification used in the present study shown on 3D computed tomography angiography (CTA) images (a) and 2D fluoroscopic angiography (FA) images of patient 14 (b). LAO left anterior oblique, $R A O$ right anterior oblique
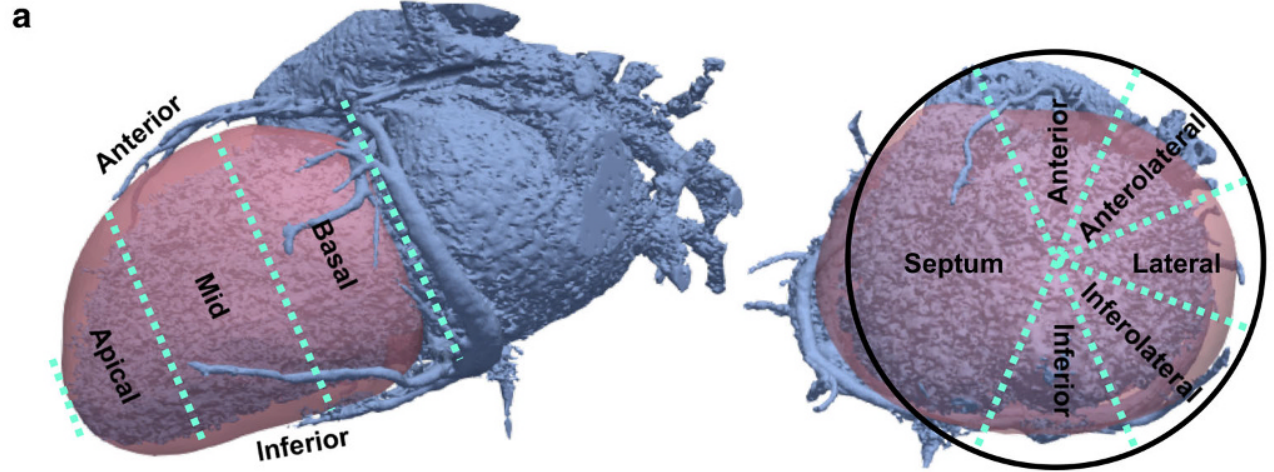

b
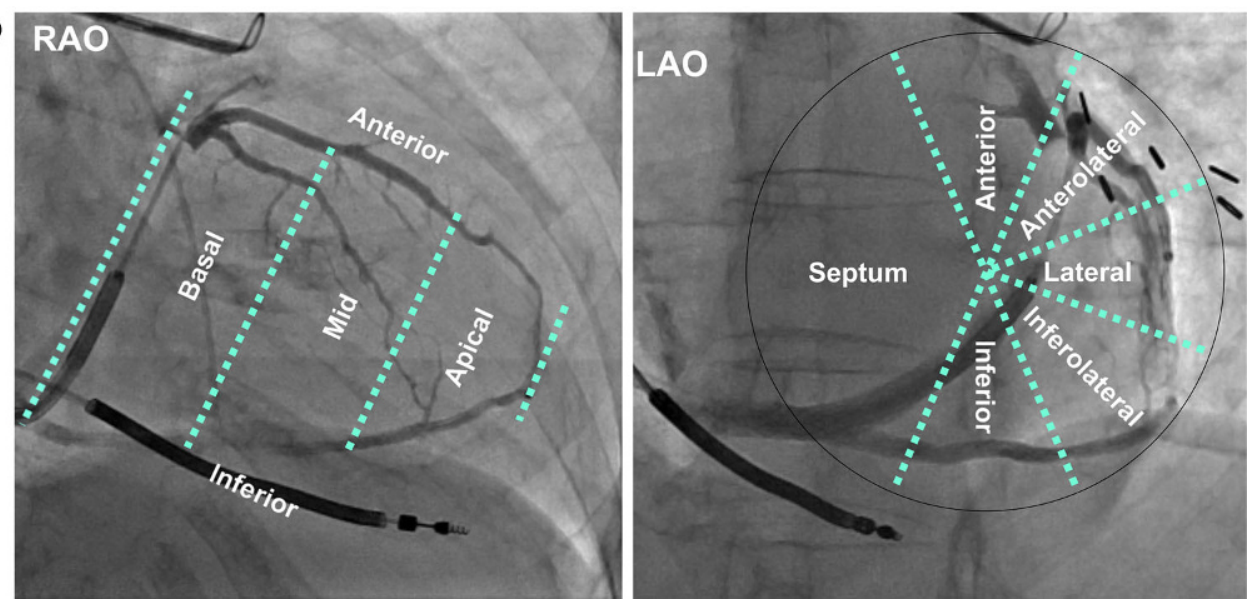

reconstructed CTA images were visually compared with those present on FA in RAO and LAO views (supplementary Fig. I).

\section{Results}

Eighteen patients planned for CRT device implantation were included (Tab. 1). Coronary venous CTA was successfully performed in all 18 patients. CRT implantation was carried out in 15 patients. In one patient CRT was cancelled due to spontaneous recovery of LV ejection fraction, resulting in no indication for CRT according to current guidelines (patient 9) [1]. One patient (patient 16, Fig. 2) was initially planned for CRT device implantation but was treated with a dual-chamber pacemaker after suboptimal coronary venous anatomy on CTA. This patient had undergone an unsuccessful CRT implantation 5 years earlier as no lateral vein (LTV) was accessible for LV-lead placement. The patient was referred for reconsideration of CRT. CTA imaging revealed two major issues for conventional LV-lead placement (Fig. 2). First, there was only a single small LTV available. Second, the CS branched into two sub-veins, further complicating cannulation of the single vein. For these reasons, CRT implantation was abandoned and the patient was treated with a dual-chamber pacemaker only instead. One patient (patient 15) died prior to the scheduled CRT implantation, unrelated to the study protocol.

In one patient (patient 5, supplementary Fig. II) CRT implantation was postponed due to the finding of a RV thrombus on CTA for which anticoagulant therapy was initiated. The patient still underwent CRT implantation 6 months post-dissolution of the RV thrombus on repeat CTA.

A standard left-sided CRT implantation approach by entering the left subclavian or cephalic vein and advancing the CRT leads to the right atrium through the left innominate vein was used in 13 patients. A right-sided implantation approach was carried out in the remaining 2 patients (numbers 4 and 10, Fig. 3) because of a persistent left-sided superior vena cava (SVC) with no communicating left innominate vein to reach the right-sided SVC from the left subclavian vein. In patient 10 , the presence of a persistent left-sided SVC was known beforehand, and in patient 4 it was found on coronary venous CTA. Both patients had dilatation of the CS as a consequence of the persistent SVC.

An overview of the coronary veins present on CTA and FA with final LV-lead placement per individual patient is provided in Fig. 4. In one patient (patient 4, Fig. 3a) the LV lead was initially targeted towards the anterolateral vein, but cannulation did not succeed due to a steep angulation at the ostium. After multiple failed attempts, the LV lead was 
Table 1 Patient characteristics

\begin{tabular}{lc}
\hline Patient characteristics $(n=18)$ & \\
\hline Demographics & $69 \pm 9$ \\
Age (years) & $13(72 \%)$ \\
Male $(n)$ & $29 \pm 5$ \\
BMI $\left(\mathrm{kg} / \mathrm{m}^{2}\right)$ & $12(67 \%)$ \\
Ischaemic cardiomyopathy $(n)$ & $7 / 11$ \\
NYHA (II/III) & \\
CMR LV function & $27 \pm 9$ \\
LVEF $(\%)$ & $302 \pm 86$ \\
EDV (ml) & $234 \pm 93$ \\
ESV (ml) & $69 \pm 18$ \\
SV (ml) & \\
ECG characteristics & $14(78 \%)$ \\
Sinus rhythm $(n)$ & $3(17 \%)$ \\
Atrial fibrillation $(n)$ & $1(6 \%)$ \\
Paced rhythm $(n)$ & $145 \pm 24$ \\
QRS duration (ms) & $9(50 \%)$ \\
LBBB $(n)$ &
\end{tabular}

$B M I$ body mass index, $C M R$ cardiac magnetic resonance, $E C G$ electrocardiogram, $E D V$ end-diastolic volume, $E S V$ end-systolic volume, $L B B B$ left bundle branch block, $L V$ left ventricular, $L V E F$ LV ejection fraction, $N Y H A$ New York Heart Association, $S V$ stroke volume

finally placed in the inferior vein (IV). A sharp angulation of the initial target vein (inferolateral vein (ILV)) was also the case in patient 3 (supplementary Fig. III), where cannulation failed and the LV lead was finally cannulated into the IV and advanced up to the ILV through an anastomosis.

The number of veins per patient ranged between 2 and 5. Coronary venous anatomy was extremely limited to only two veins in patient 10 (Fig. 3b). A total of 62 veins from 16 patients with both CTA and angiographic images were visualised. Eighty-five percent (53/62) of the veins were visualised on CTA as well as FA, while $10 \%(6 / 62)$ were visualised on CTA only, and 5\% (3/62) were visible on FA only. A total of 22 veins were present on the lateral or inferolateral wall, of which $95 \%$ (21/22) were visualised by CTA. Veins visualised on CTA only were most often located inferiorly (4/6), while veins visualised on FA only were small and located anterolaterally (2/3) or inferolaterally $(1 / 3)$.

\section{Discussion}

This study illustrates the additive value of coronary venous imaging by CTA prior to CRT implantation by elucidating clinical cases and by comparing coronary venous anatomy visualisation by CTA with FA.

In patients with both CTA and FA data, there was a high concordance ( $85 \%$ ) between coronary venous CTA and FA.
Interestingly, $10 \%$ of the veins were visualised by CTA but not on FA, indicating that CTA may even have the capability of identifying veins that are not shown by FA. Two-thirds of the veins missed by FA were, however, located inferiorly, which is usually not the preferred site of LV-lead placement. In contrast, $95 \%$ of the ILV or LTV (the preferred veins in CRT) were visualised by CTA. Five per cent of the veins were only visible on FA and therefore missed on CTA. These three veins were, however, small and not suitable for LV-lead placement.

\section{The relevance of coronary venous anatomy visualisation in CRT}

In about a quarter of our patients, pre-procedural cardiac venous CTA had a major impact on the CRT implantation approach. In two patients, a right-sided implantation was carried out due to a persistent left-sided SVC with no left innominate vein. In one patient, implantation was postponed for 6 months due to a RV thrombus, and in another patient, a dual-chamber pacemaker was implanted instead of CRT due to unfavourable coronary venous anatomy.

Coronary venous CTA was particularly useful for the implanting physician by visualising the coronary veins in 3D prior to CRT implantation, allowing anticipation of patientspecific anatomy. The number and trajectory of coronary veins was highly variable in our patients; in two of them, for instance, initial target veins were inaccessible during implantation due to steep angulations at the ostium, and in one patient only two coronary veins were present with limited options for conventional LV-lead placement. A high variability in coronary venous anatomy was also seen in coronary venous CTA imaging in 121 perfusion-fixed postmortem human hearts [7] Remarkably, in $29 \%$ of the hearts no coronary vein was present in the inferolateral region, which is often the site of late activation and subsequently a sweet-spot for LV-lead placement in CRT.

Besides CTA, coronary venous anatomy can also be visualised pre-procedurally in a subset of CRT candidates who are already scheduled for a routine coronary angiography using venous phase coronary angiography [8] Coronary venous anatomy visualisation may be useful in identifying those patients with suboptimal coronary venous anatomy and may potentially simplify the CRT implantation procedure, as it may reduce the need for extensive fluoroscopic coronary venous angiograms. In addition to CRT, evaluation of the coronary venous anatomy may also be relevant for other procedures, including radiofrequency ablation [9] or cell therapy [10]. 
a
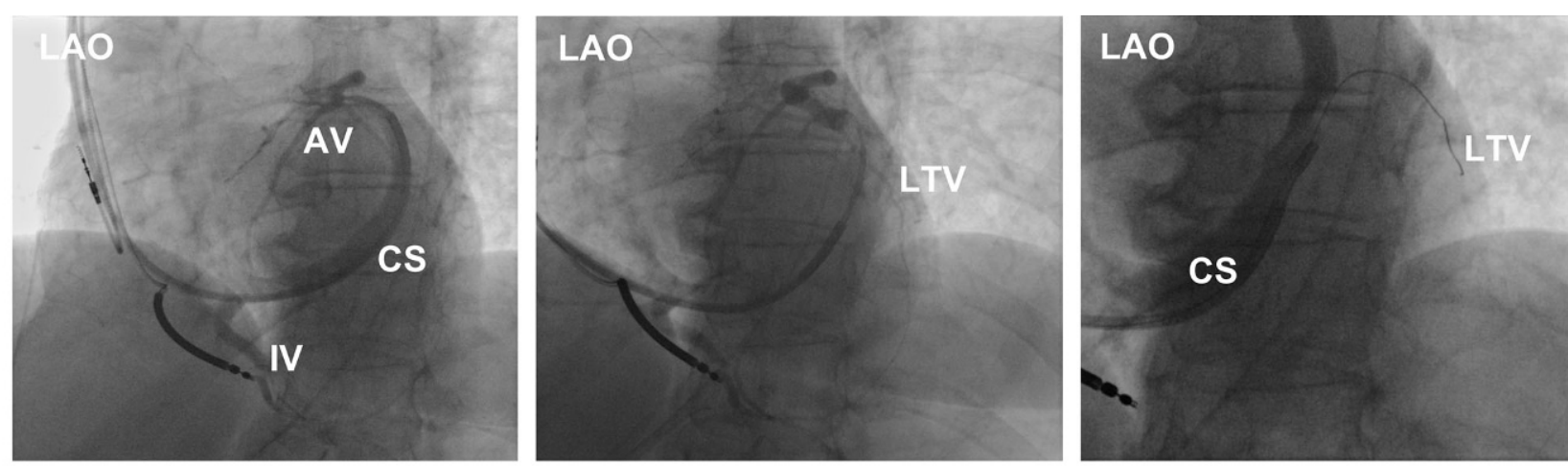

b
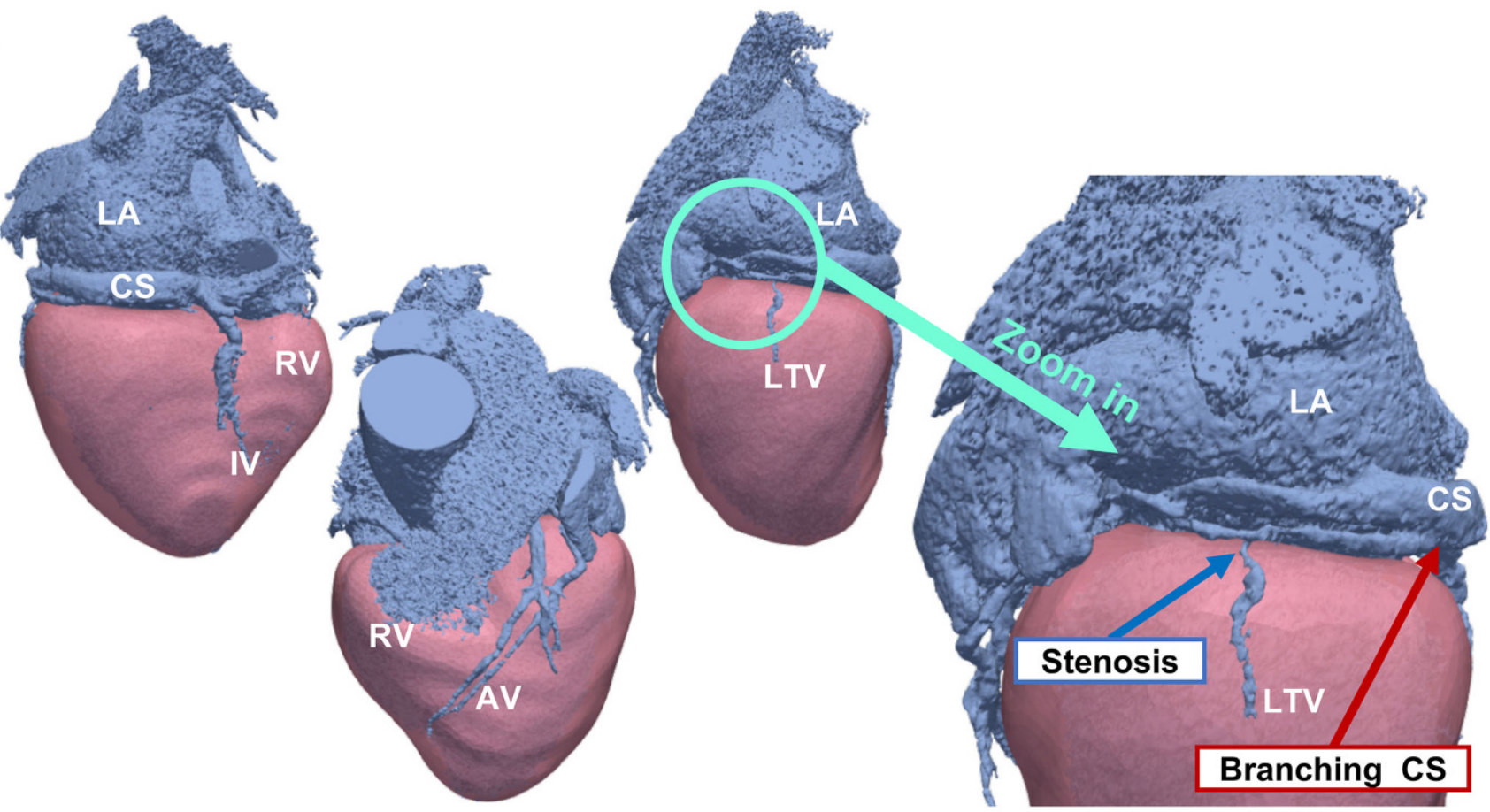

C
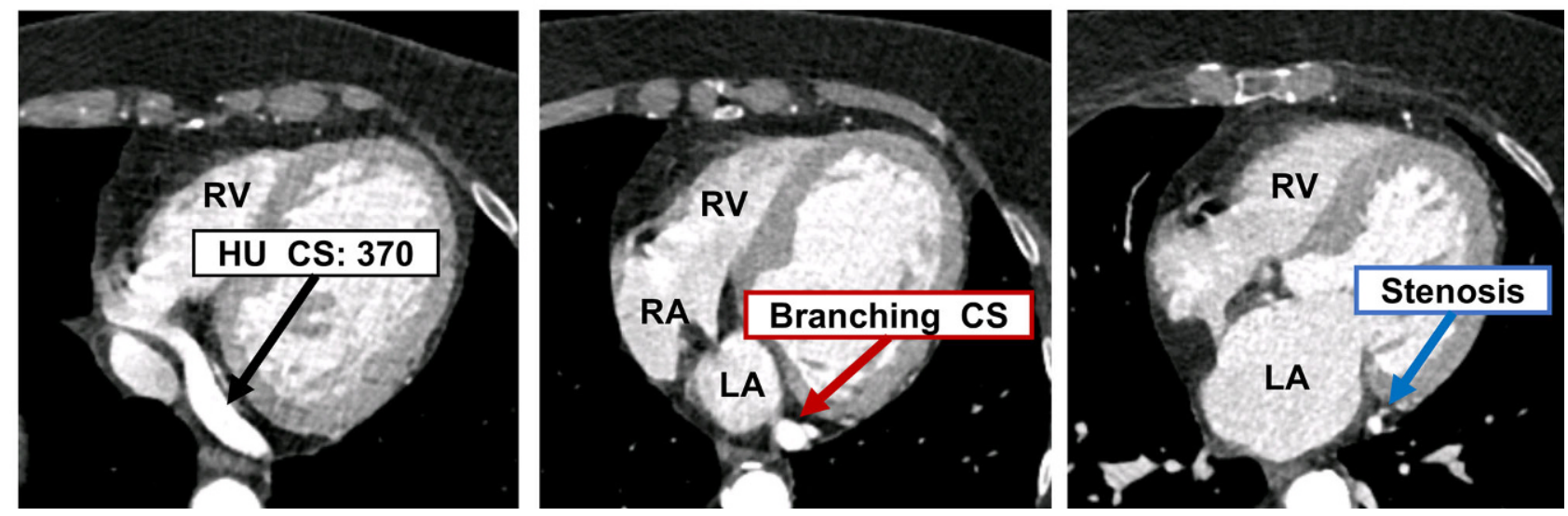

Fig. 2 A 78-year-old woman (patient 16) who had undergone an unsuccessful cardiac resynchronisation therapy implantation 5 years earlier as no lateral vein on FA (a) was accessible for left ventricular lead placement. CTA in 3D (b) and 2D (c) revealed a single small and stenotic LTV and CS branching. $A V$ anterior vein, $C S$ coronary sinus, $I V$ inferior vein, $L A$ left atrium, $L A O$ left anterior oblique, $L T V$ lateral vein, $R A$ right atrium, $R V$ right ventricle 
Fig. 3 CTA and FA of a 77-year-old woman (patient 4 , a) and a 49-year-old woman (patient 10, b) with a left-sided persistent superior vena cava (SVC, red arrow) and CS dilatation. Note the sharply angulated vein (white arrow) in patient 4 (a) and the limited coronary venous anatomy in patient 10 (b). $A L V$ anterolateral vein, $A V$ anterior vein, $C S$ coronary sinus, ILV inferolateral vein, $I V$ inferior vein, $L A$ left atrium, $L A O$ left anterior oblique, $R A$ right atrium, $R A O$ right anterior oblique, $R V$ right ventricle
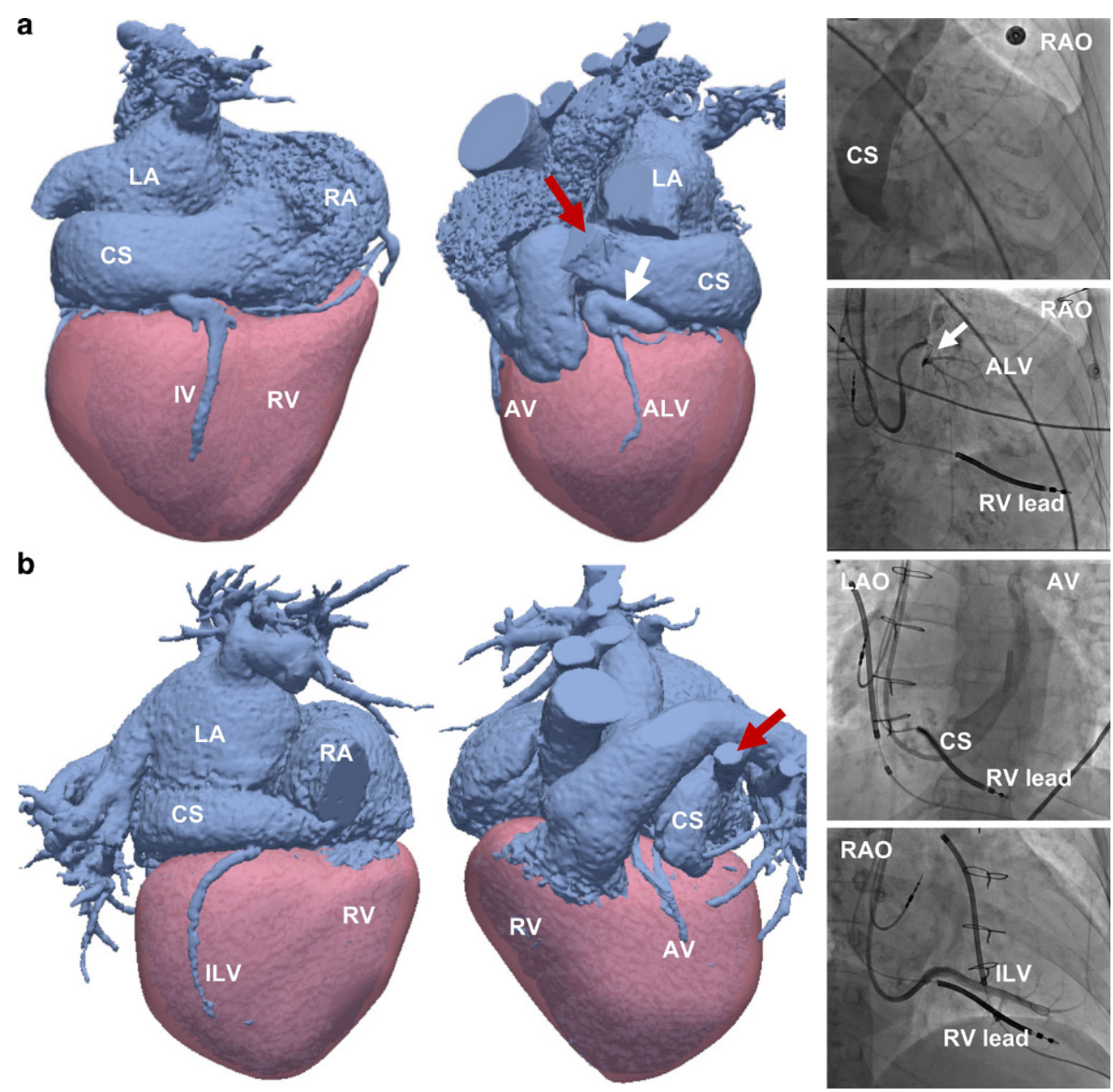

\section{Moving towards a tailored coronary venous CTA protocol}

The coronary venous anatomy has been assessed by a 4MDCT scanner and compared with FA in a small study of seven patients [11] but still remains an underexposed area of research compared to the arterial system. The greatest challenge for coronary venous imaging is determining optimal delay for CTA acquisition after the administration of CM, whilst still keeping the total iodine load low. Recent coronary arterial CTA studies demonstrate that CM protocols tailored to the individual patient lead to robust enhancement patterns and reduction of the amount of CM used [12, 13]. CRT patients have low LV function, resulting in prolonged duration for $\mathrm{CM}$ to reach the coronary system. Contrast medium furthermore circulates in the body, is diluted by blood, and the bolus disperses as it moves downstream in the circulatory system. The coronary veins are located more distal from the injection site in comparison with the arteries and therefore encounter more dilution. In addition, CM-enhanced blood recirculates and may contribute to the overall pattern of contrast enhancement achieved on CTA imaging.
In our coronary venous CTA protocol contrast and thus high attenuation of the coronary veins was accomplished by accurate timing of CM administration ( $+10 \mathrm{~s}$ for the $\mathrm{CS} ;+20 \mathrm{~s}$ for the ascending aorta) using a relatively low dose of $\mathrm{CM}$ (26.6g iodine in total).

\section{Future prospects}

Stimulating the correct LV pacing site remains a critical factor for response to CRT. The need for sites of late activation remote from scarring, acceptable pacing thresholds without phrenic nerve stimulation, limited and suboptimal coronary venous anatomy places major constraints on the available LV pacing sites. In case of limited coronary venous anatomy or extensive scarring around the veins, alternative approaches for biventricular pacing could be considered, including endocardial pacing $[14,15]$, trans-septal pacing [16] or epicardial pacing using a minimally invasive video-assisted thoracic surgical approach [17].

In this study, we focussed primarily on coronary venous anatomy imaging. However, additional advantage could be taken from CT in the context of CRT by incorporating 


\section{Coronary veins}

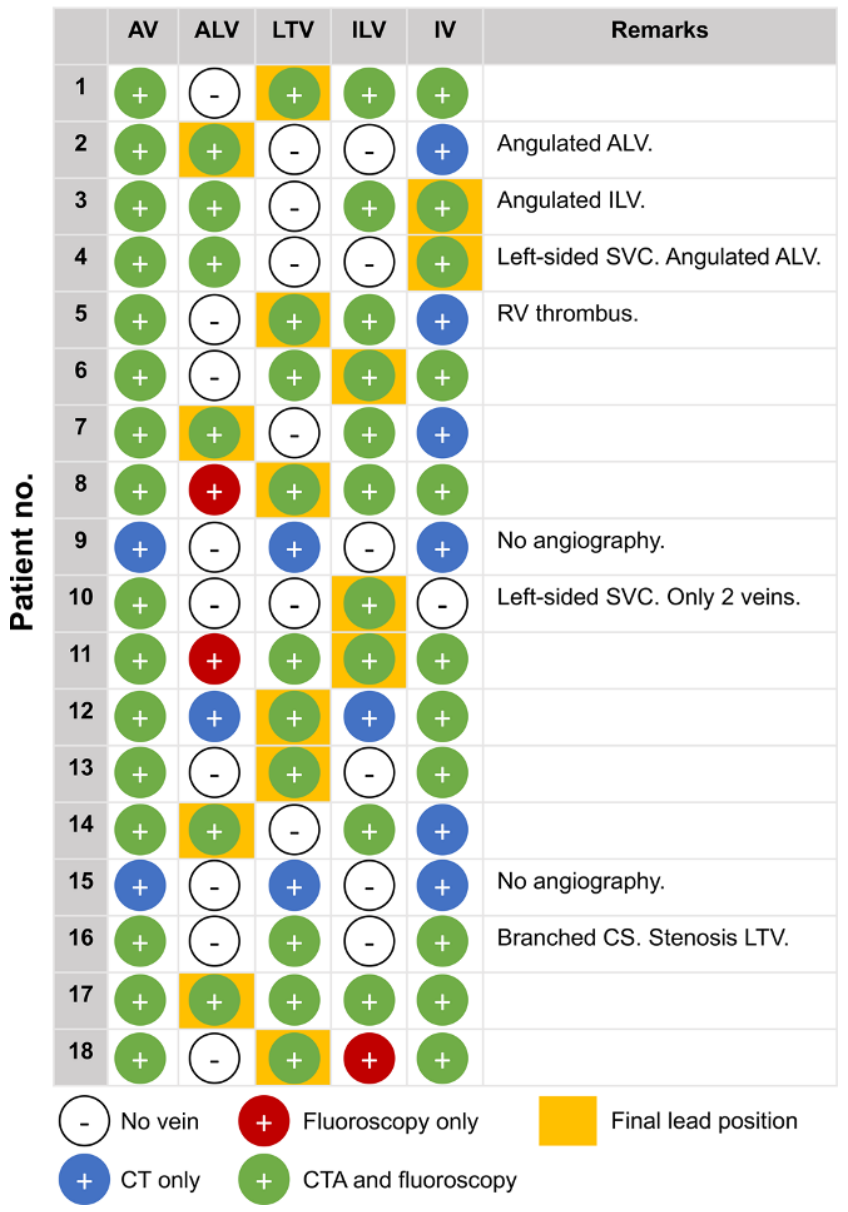

Fig. 4 Coronary veins on CTA and FA with final left ventricular lead position. $A L V$ anterolateral vein, $A V$ anterior vein, $C S$ coronary sinus, $I L V$ inferolateral vein, $I V$ inferior vein, $L T V$ lateral vein, $R V$ right ventricle, $S V C$ superior vena cava

wall thinning, wall motion [18] or delayed enhancement as a metric of myocardial scarring. Imaging of coronary venous anatomy together with dyssynchrony metrics and myocardial scarring using only CT prior to CRT was very recently performed in 54 patients by Truong et al. [19]. This is particularly relevant in patients with already existing pacing systems [20] in which CMR is contra-indicated. An additional potential advantage of CTA may be visualisation of the phrenic nerve [21] in relation to the coronary venous anatomy, to avoid phrenic nerve stimulation around the LV lead.

\section{Limitations}

This is a single-centre study using a limited number of patients. However, coronary venous CTA already impacted on a substantial percentage of the CRT implantations, indicating that CTA prior to CRT may be of important clinical value. CM should be used with caution in patients with severe kidney dysfunction; however CM doses were kept low.

\section{Conclusion}

Visualisation of the coronary venous anatomy by CTA is representative of the anatomy recorded intra-procedurally under FA and has additive value for CRT implantation. CTA allows patient-specific anticipation of coronary venous anatomy and determination of relevant LV-lead pacing sites, making it a diagnostic of high potential in CRT.

Conflict of interest U.C. Nguyên received a Kootstra Talent Fellowship research grant from Maastricht University and was additionally funded by a research grant from MS from the Dutch Heart Foundation (grant \#2015T61). M.J.M. Cluitmans is supported by the Hein Wellens Fonds and is part-time employed by Philips. J.E. Wildberger received institutional grants from Agfa, Bayer, GE, Phillips, and Siemens and speaker fees from Bayer and Siemens. F.W. Prinzen received research grants from Medtronic, Abbott, LivaNova, Biosense Webster, MSD, and Biotronik. K. Vernooy received research grants from Medtronic and Abbott. J.G.L.M. Luermans, M. Strik, C.B. de Vos, B.L.J.H. Kietselaer and C. Mihl declare that they have no competing interests.

Ethical standards All procedures performed in studies involving human participants were in accordance with the ethical standards of the institutional and/or national research committee and with the 1975 Helsinki declaration and its later amendments or comparable ethical standards. Informed consent was obtained from all individual participants included in the study.

Open Access This article is distributed under the terms of the Creative Commons Attribution 4.0 International License (http:// creativecommons.org/licenses/by/4.0/), which permits unrestricted use, distribution, and reproduction in any medium, provided you give appropriate credit to the original author(s) and the source, provide a link to the Creative Commons license, and indicate if changes were made.

\section{References}

1. European Society of C, European Heart Rhythm A, Brignole M, Auricchio A, Baron-Esquivias G, et al. 2013 ESC guidelines on cardiac pacing and cardiac resynchronization therapy: the task force on cardiac pacing and resynchronization therapy of the European Society of Cardiology (ESC). Europace. 2013;15(8):1070-118. Developed in collaboration with the European Heart Rhythm Association (EHRA).

2. Rademakers LM, van Kerckhoven R, van Deursen CJ, et al. Myocardial infarction does not preclude electrical and hemodynamic benefits of cardiac resynchronization therapy in dyssynchronous canine hearts. Circ Arrhythm Electrophysiol. 2010;3:361-8.

3. Khan FZ, Virdee MS, Palmer CR, et al. Targeted left ventricular lead placement to guide cardiac resynchronization therapy: the TARGET study: a randomized, controlled trial. J Am Coll Cardiol. 2012;59:1509-18.

4. Bakos Z, Markstad H, Ostenfeld E, Carlsson M, Roijer A, Borgquist R. Combined preoperative information using a bullseye plot from speckle tracking echocardiography, cardiac CT scan, and MRI scan: targeted left ventricular lead implantation in patients receiving cardiac resynchronization therapy. Eur Heart J Cardiovasc Imaging. 2014;15:523-31. 
5. Blendea D, Shah RV, Auricchio A, et al. Variability of coronary venous anatomy in patients undergoing cardiac resynchronization therapy: a high-speed rotational venography study. Heart Rhythm. 2007;4:1155-62.

6. Van de Veire NR, Schuijf JD, De Sutter J, et al. Non-invasive visualization of the cardiac venous system in coronary artery disease patients using 64-slice computed tomography. J Am Coll Cardiol. 2006;48:1832-8.

7. Spencer JH, Larson AA, Drake R, Iaizzo PA. A detailed assessment of the human coronary venous system using contrast computed tomography of perfusion-fixed specimens. Heart Rhythm. 2014;11:282-8.

8. Mischke K, Knackstedt C, Muhlenbruch $\mathrm{G}$, et al. Imaging of the coronary venous system: retrograde coronary sinus angiography versus venous phase coronary angiograms. Int J Cardiol. 2007;119:339-43.

9. Sanders P, Jais P, Hocini M, Haissaguerre M. Electrical disconnection of the coronary sinus by radiofrequency catheter ablation to isolate a trigger of atrial fibrillation. J Cardiovasc Electrophysiol. 2004;15:364-8.

10. Gathier WA, van Ginkel DJ, van der Naald M, van Slochteren FJ, Doevendans PA, Chamuleau SAJ. Retrograde coronary venous infusion as a delivery strategy in regenerative cardiac therapy: an overview of preclinical and clinical data. J Cardiovasc Transl Res. 2018;11:173. https://doi.org/10.1007/s12265-018-9785-1.

11. Muhlenbruch G, Koos R, Wildberger JE, Gunther RW, Mahnken $\mathrm{AH}$. Imaging of the cardiac venous system: comparison of MDCT and conventional angiography. AJR Am J Roentgenol. 2005;185: 1252-7.

12. Mihl C, Kok M, Altintas S, et al. Evaluation of individually body weight adapted contrast media injection in coronary CT-angiography. Eur J Radiol. 2016;85:830-6.

13. Mihl C, Kok M, Wildberger JE, et al. Coronary CT angiography using low concentrated contrast media injected with high flow rates: feasible in clinical practice. Eur J Radiol. 2015;84:2155-60.
14. Bracke FA, van Gelder BM, Dekker LR, Houthuizen P, Ter Woorst JF, Teijink JA. Left ventricular endocardial pacing in cardiac resynchronisation therapy: Moving from bench to bedside. Neth Heart J. 2012;20:118-24.

15. Strik M, Rademakers LM, van Deursen CJ, et al. Endocardial left ventricular pacing improves cardiac resynchronization therapy in chronic asynchronous infarction and heart failure models. Circ Arrhythm Electrophysiol. 2012;5:191-200.

16. Mafi-Rad M, Luermans JG, Blaauw Y, et al. Feasibility and acute hemodynamic effect of left ventricular septal pacing by transvenous approach through the interventricular septum. Circ Arrhythm Electrophysiol. 2016;9:e3344.

17. Schouwenburg JJ, Klinkenberg TJ, Maass AH, Mariani MA. Video-assisted thoracic placement of epicardial leads. J Card Surg. 2014;29:286-9.

18. Behar JM, Rajani R, Pourmorteza A, et al. Comprehensive use of cardiac computed tomography to guide left ventricular lead placement in cardiac resynchronization therapy. Heart Rhythm. 2017; 14:1364-72.

19. Truong QA, Szymonifka J, Picard MH, et al. Utility of dual-source computed tomography in cardiac resynchronization therapy-DIRECT study. Heart Rhythm. 2018. https://doi.org/10.1016/j.hrthm. 2018.03.020

20. Ter Horst IA, Kuijpers Y, van 't Sant J, Tuinenburg AE, Cramer MJ, Meine M. "Are CRT upgrade procedures more complex and associated with more complications than de novo CRT implantations?" A single centre experience. Neth Heart J. 2016;24:75-81.

21. Wang YJ, Liu L, Zhang MC, Sun H, Zeng H, Yang P. Imaging of pericardiophrenic bundles using multislice spiral computed tomography for phrenic nerve anatomy. J Cardiovasc Electrophysiol. 2016;27:961-71. 


\title{
Advertisement placed here.
}

\author{
SCS bohn \\ stafleu \\ CL van loghum \\ Springer Media
}

Houten 2018 


\title{
Advertisement placed here.
}

\author{
SCS bohn \\ stafleu \\ CL van loghum \\ Springer Media
}

Houten 2018 


\title{
Advertisement placed here.
}

\author{
SCS bohn \\ stafleu \\ CL van loghum \\ Springer Media
}

Houten 2018 


\title{
Advertisement placed here.
}

\author{
SCS bohn \\ stafleu \\ CL van loghum \\ Springer Media
}

Houten 2018 